\title{
Las capacidades tecnológicas para la innovación en empresas de manufactura
}

Technological Capabilities for Innovation in Manufacturing Companies

\section{As capacidades tecnológicas para a inovação em empresas de manufatura}

\author{
Arturo García Velázquez \\ Daniel Pineda Domínguez ${ }^{\star \star}$ \\ María Antonieta Andrade Vallejo ${ }^{* \star *}$
}

Fecha de recibido: 22 de diciembre de 2014

Fecha de aprobado: 28 de abril de 2015

Doi: dx.doi.org/10.12804/rev.univ.empresa.29.2015.11

Para citar este artículo: García Velázquez, A., Pineda Domínguez, D., \& Andrade Vallejo, M. A. (2015). Las capacidades tecnológicas para la innovación en empresas de manufactura. Universidad \& Empresa, 1729), 257-278. Doi: dx.doi.org/10.12804/rev.univ.empresa.29.2015.11

* Maestro en Ciencias en Administración de Negocios de la Escuela Superior de Comercio y Administración (ESCA) del Instituto Politécnico Nacional (IPN). (Distrito Federal, México). Estudiante del Doctorado en Ciencias Administrativas (ESCA) del (ESCA). (Distrito Federal, México). Correo electrónico: arturogarciavel@yahoo.com.mx

** Doctor en Ciencias Administrativas de la Escuela Superior de Comercio y Administración (ESCA) del Instituto Politécnico Nacional (IPN). (Distrito Federal, México) Catedrático investigador de la esca, Sto. Tomás (Distrito Federal, México). Correo electrónico: danpin07@yahoo.com.mx

*** Doctora en Ciencias Administrativas de la Escuela Superior de Comercio y Administración (ESCA) del Instituto Politécnico Nacional (IPN). (Distrito Federal, México). Doctora en Ciencias de la Educación, Colegio de Estudios de Posgrado de la Ciudad de México (Edo. de México). Maestría en Ciencias Administrativas, Unidad Profesional Interdisciplinaria de Ingeniería y Ciencias Sociales Administrativas (UPIICSA) del IPN. Catedrática investigadora de la ESCA, Sto. Tomás. Correo electrónico: mandradev@ipn.mx 


\section{RESUMEN}

Las capacidades tecnológicas son habilidades requeridas para un uso efectivo del conocimiento tecnológico. Permiten que las empresas de manufactura puedan innovar, un requisito básico para competir de manera efectiva en los mercados. El objetivo de este trabajo de investigación fue analizar los factores que relacionan las capacidades tecnológicas con la innovación. Para ello se hizo un análisis de la literatura, considerando los aportes de múltiples autores relevantes. Se contrastaron, además, argumentos existentes sobre empresas en economías maduras con algunos que consideran la realidad de organizaciones en economías emergentes. Se encontró que las capacidades tecnológicas son un factor determinante de innovación, pero que estas varían, en particular, en función del nivel de desarrollo de los países.

Palabras clave: capacidades tecnológicas, conocimiento, empresas de manufactura, innovación.

\section{ABSTRACT}

The technological capabilities are abilities required for the effective use of technological knowledge. They allow manufacturing companies to innovate, which is a basic requirement to compete effectively in markets. The aim of this research was to analyze the factors that put in relation technological capacities with innovation. For this purpose, a literature review, that took into consideration the contributions of many important authors, was carried out. In addition, existing arguments regarding companies in mature economies were contrasted with others that consider the reality of organizations in emerging economies. It was found that technological capabilities are a determining factor for innovation, but that they vary, in particular, as a function of the countries' development level.

Keywords: Technological capabilities, knowledge, manufacturing companies, Innovation.

\section{RESUMO}

As capacidades tecnológicas são habilidades requeridas para um uso efetivo do conhecimento tecnológico. Permitem que as empresas de manufatura possam inovar, um requisito básico para competir de maneira efetiva nos mercados. O objetivo deste trabalho de pesquisa foi analisar os fatores que relacionam as capacidades tecnológicas com a inovação. Para isto, se fez uma análise da literatura considerando os aportes de múltiplos autores relevantes. Contrastaram-se, além, argumentos existentes sobre empresas em economias maduras com alguns que consideram a realidade de organizações em economias emergentes. Encontrou-se que as capacidades tecnológicas são um fator determinante de inovação, mas que estas variam, em particular, em função do nível de desenvolvimento dos países. Palavras-chave: capacidades tecnológicas, conhecimento, empresas de manufatura, inovação. 


\section{INTRODUCCIÓN}

Los constantes cambios de la economía mundial imperante en la actualidad han llevado a aumentar la competencia entre las empresas. Ello explica que algunas economías maduras tengan un gran poder en el mercado global. Esta situación se relaciona con la demanda de ventajas estratégicas en innovación en materia de procesos y productos, lo que se consigue, entre otras, a través del desarrollo de capacidades tecnológicas. Por otro lado, las debilidades de las empresas manufactureras y su escaso desarrollo tecnológico son consecuencia de la mínima innovación, así como de su bajo y desordenado crecimiento en la incorporación de tecnologías de diseño y manufactura, lo que dificulta que se establezcan criterios tecnológicos para el apoyo a estas empresas.

La literatura científica considera que las empresas buscan desarrollar nuevas capacidades con el fin de adaptarse rápidamente a los requerimientos del mercado, ya que la necesidad de acumular y renovar capacidades permite administrar el conocimiento, la innovación y el aprendizaje, además de intensificar la competencia entre las empresas (Dutrenit \& Arias, 2002).

Las capacidades tecnológicas son un determinante para la innovación en las empresas de manufactura, ya que permean diferentes aspectos del desarrollo de la organización, pero, principalmente, en este tipo de sector $\mathrm{y}$, en particular, en las pequeñas y medianas empresas. Estas usualmente tienen dificultades para realizar innovación de procesos y productos en áreas tan sensibles como el diseño de ingeniería, el diseño detallado, la organización de la producción y el mejoramiento de las actividades de manufactura avanzada.

El comportamiento de las empresas está, entre otros factores, en función de una serie de características que varían de acuerdo con su tamaño (microempresas, pequeñas, medianas y grandes). También cambia en función de su origen (nacionales, extranjeras o mixtas) y su capital (si son privadas, públicas o mixtas). La innovación depende de estas circunstancias, así como de los dominios tecnológicos que se dan en las empresas. Esta depende también del apoyo que brindan los gobiernos y de la disposición de participar de las empresas en la adquisición de los cuatro recursos cuya consideración, de acuerdo con Barney (1991), es fundamental. Estos son: humanos, financieros, materiales y tecnológicos (Barney \& Clark, 2007).

No son muchos los investigadores que relacionan el desarrollo de las 
capacidades tecnológicas con la innovación en los contextos empresariales, aunque algunos lo mencionan cuando aluden a las economías maduras. Bell y Pavitt (1995) se identifican como los autores que tocaron este tema por primera vez. Ellos resaltaron la importancia de las capacidades tecnológicas en los países con economías maduras. Por la profundidad de sus aportaciones, estos investigadores sirvieron como punto de referencia para contrastar las investigaciones que surgieron posteriormente.

Chung y Swink (2009), unos de los aportantes posteriores, sostuvieron que para desarrollar capacidades tecnológicas las empresas de economías emergentes deben seguir los mismos patrones que tomaron en cuenta las empresas en las economías maduras, aunque, por supuesto, considerando sus propias características y ponderando las diferencias entre unas y otras. Esto para poder adaptar de manera adecuada los modelos a sus particularidades y necesidades básicas de desarrollo.

Las capacidades tecnológicas son habilidades esenciales para la innovación de las empresas de manufactura en las economías emergentes. Estas, en la actualidad, son consideradas un recurso importante para la competitividad. El objetivo de esta investigación, considerando lo anterior, es analizar los factores que relacionan las capacidades tecnológicas con la innovación, los cuales requieren tomar en cuenta las empresas de manufactura para competir en los mercados. Ello se hace mediante la revisión de la literatura especializada y tomando en cuenta qué tanto se sabe y aborda el tema para ubicar hallazgos, vacíos y limitaciones con el fin de proponer nuevas pistas y líneas de investigación. El documento está estructurado en cuatro secciones: la revisión de la literatura, la metodología, la puesta en relieve de los resultados más sobresalientes y, finalmente, la discusión y las conclusiones.

\section{REVISIÓN DE LA LITERATURA}

Las constantes transformaciones de la economía mundial y los cambiantes requerimientos de la globalización, conducen al uso, creación y adaptación de nuevas tecnologías y generan que los países tengan la necesidad de transformarse para poder enfrentar las nuevas exigencias que imponen los mercados. En este contexto, las empresas en economías emergentes requieren poder ser capaces de asimilar, usar, adaptar, cambiar, crear y mejorar las tecnologías. Esto dependiendo del tipo de sector al que pertenecen y de la realidad particular que enfrentan. 
La Organización para la Cooperación y el Desarrollo Económico (OECD, 2005) clasifica los sectores industriales de acuerdo con su intensidad tecnológica. Estos, en efecto, tienen características diferenciadas en razón del perfil de las empresas con respecto a la capacidad tecnológica de los países emergentes, los que, a su vez, se clasifican considerando su sistema de producción, procesos y productos, indicadores que sirven para medir dicha intensidad tecnológica, la cual puede ser baja, media-baja, media y alta. Esto en el contexto considerado.

En los años noventa, gracias fundamentalmente a los aportes de Bell \& Pavitt (1995), fue posible clasificar a las empresas según la tecnología aplicada dentro de su sector, considerando que un sector no necesariamente tiene o exige el mismo nivel de capacidad tecnológica que otro. Las empresas de manufactura, por su parte, se evalúan de acuerdo con su tamaño, origen, capital (inversión nacional y extranjera). Se considera que los cambios tecnológicos se originan en los sectores industriales que dan forma a los sistemas económicos, tales como las industrias extractivas, las mineras, el petróleo, la transformación, la manufacturera, etc., así como en la maquinaria y los equipos que influyen de manera decisiva en los procesos y productos (Bell \& Pavitt, 1995).
Para entender la importancia de las capacidades tecnológicas, es preciso tomar en cuenta las cambiantes necesidades del mercado, las cuales presionan a las empresas a ser cada vez más innovadoras para no perder su competitividad (Madhok, Li, \& Priem, 2010). En efecto, el mercado y las capacidades tecnológicas afectan el desempeño de las empresas. Estas, para poder adaptarse y responder a las demandas actuales requieren diferentes funciones y niveles de tecnología, aunque, simultáneamente, deben comprometerse a desarrollar y construir esfuerzos y habilidades en el conjunto de la organización (González \& Cunha, 2012).

Según Acosta Prado, Bueno Campos \& Longo Somoza (2014) la creación y explotación del conocimiento son la fuente fundamental de las capacidades tecnológicas de las empresas. De ahí la importancia de contemplar el desarrollo, la acumulación y el uso de conocimientos dentro de la organización, la asimilación de los elementos necesarios provenientes del exterior de la misma, la tecnológica individual, la tecnología organizacional, los comportamientos y las habilidades de los individuos, las habilidades de la organización y los demás comportamientos que tienen algún tipo de influencia dentro y fuera de la empresa. 
Las empresas son cada vez más consideradas como motores esenciales del desarrollo regional y pilares de las economías emergentes, en particular para la generación de empleos técnicos especializados susceptibles a los cambios en la economía global. Estas dependen de manera creciente de redes de conocimiento, así como de la iniciativa empresarial y el aprendizaje interactivo y creativo. Estos factores son necesarios para lograr la innovación y establecer vínculos productivos entre las empresas y los organismos gubernamentales, así como entre estas y el mundo académico (Etzkowitz, 2008; Gibbs \& Humphries, 2009; Yokakul \& Zawdie, 2010). La fuente de la ventaja competitiva ha cambiado entonces para muchas empresas de manufactura, ya que su penetración en el mercado está basada de manera importante en sus propios recursos tecnológicos y en el desarrollo de sus capacidades de respuesta.

Ellonen y Jantunen (2011) mencionan también el papel que las capacidades dinámicas tienen en el desarrollo de las capacidades operativas relacionadas con la innovación. Las capacidades dinámicas son por naturaleza procesos y prácticas que promueven el cambio (Augier \& Teece, 2008; Teece, 2008; Teece, Pisano, \& Shuen, 1997; Winter, 2008). Dado que una capacidad dinámica se ve afectada por el mercado, las empre- sas deben contemplar también capacidades estratégicas de marketing. Más aún, la capacidad de identificación dinámica es una oportunidad para reconfigurar procesos de producción, con el fin de tener un efecto positivo en la comercialización sustantiva y la creación de capacidades tecnológicas (Wilden, Gudergan, \& Lings, 2011). Así, un factor clave radica hoy en privilegiar más las capacidades tecnológicas, por el impacto que tienen en toda la organización, que solo desarrollar capacidades de comercialización, cuyo efecto es muy limitado.

\subsection{Ventajas y beneficios}

Las capacidades tecnológicas pueden propiciar la innovación, a través de la inversión, la producción, el soporte y la adaptación integral de las organizaciones, aprovechando la experiencia de empresas de manufactura no solo en economías maduras, sino también en emergentes Esto puede ser producto de la tendencia hacia establecer redes de colaboración interempresarial, desarrollada con cada vez más fuerza durante los últimos años.

La ventaja principal es que desarrollar un conjunto de habilidades tecnológicas permite a las empresas fortalecer nuevas capacidades que le ayudan a adaptarse rápidamente al entorno cambiante (Bell 
$\&$ Pavit, 1995). En este punto existe una coincidencia con lo señalado por Lall (1992), quien sugiere una forma de clasificar las capacidades tecnológicas y su relación con el desempeño industrial e innovador de las empresas de manufactura, mediante una matriz que es conocida como la taxonomía de las capacidades tecnológicas.

Entender la mencionada matriz puede aportar elementos para comprender los orígenes de las diferencias que se presentan en el comportamiento entre las empresas ubicadas en países con economías maduras y las que se encuentran en economías emergentes. Estas diferencias desempeñan un papel central en la teoría del proceso evolutivo, la cual destaca las consecuencias del aprendizaje en la concepción de cambio tecnológico (Nelson \& Winter, 1982). En concepto de Pavitt (1984), estos estudios son un punto de partida que permite examinar el avance de la manufactura, utilizando la codificación de trayectorias tecnológicas, la cual incluye una estimación de las capacidades tecnológicas de las empresas.

Por otro lado, diversos estudios refieren la existencia de una desventaja que se presenta en los países emergentes que carecen de capacidades tecnológicas. Esto limita sus posibilidades de innovar y obliga a que las empresas traten de conocer el desarrollo tecnológico de las economías maduras (Dutrenit \& Arias, 2002). Mediante esta premisa, Figueiredo (2004) menciona que las empresas emergentes deben recurrir al aprendizaje tecnológico para conseguir las capacidades tecnológicas. Esto a fin de lograr desarrollo tecnológico e innovación a partir de las experiencias exitosas vividas por las economías maduras.

Pese a lo anterior, se han identificado una serie de beneficios que derivan de las capacidades tecnológicas. Estos pueden ser diversos y presentarse en la forma de desarrollo económico y social, impacto en el mercado, generación de capital humano, entre otros. Esto depende del proyecto involucrado.

Zhou y Wu (2010) han identificado los beneficios más comunes de las capacididades tecnologicas. En orden de importancia se encuentran la posibilidad de diseñar una estrategia adecuada e integral, la flexibilidad para la innovación de procesos y productos y la identificación de en dónde asignar los recursos disponibles (Combs, Ketchen, Ireland, \& Webb, 2011), toda vez que las empresas dependen cada vez más del uso óptimo de estos para poder innovar. En consonancia con ello, Benner y Tushman (2003) refieren que las empresas deben invertir de manera 
decidida en la construcción de capacidades tecnológicas. Esto para desarrollar destrezas y habilidades a fin de utilizar diversos recursos adecuadamente y saber cómo hacer las cosas. Hoy se considera que, en la medida en que las empresas acumulan experiencias, conocimientos y autoaprendizaje, logran aprovechar más sus habilidades.

Trabajos como los de Dutrenit y Capdevielle (1993) establecen que un primer paso para el desarrollo de las capacidaes tecnológicas es identificar qué habilidades son necesarias para innovar y poder así hacer investigación y desarrollo en tecnologias blandas y duras. Esto mediante el análisis del perfil tecnológico del país y de su dinámica innovadora. Otro beneficio que es posible alcanzar se da cuando las compañías llegan a mantener y adaptar tecnologías a los contextos competitivos. Estas ganan y generan conocimiento $\mathrm{y}$ hacen pequeñas innovaciones que son necesarias para su funcionamiento y desarrollo económico (Reichert, Beltrame, Corso, Trevisan, \& Zawislak, 2011).

\subsection{Sobre el concepto de capacidades tecnológicas}

En un mundo global, y en una era en donde se habla de la economía del conocimiento (Schilirò, 2012), se ha ido abordando cada vez más el tema de las capacidades tecnológicas. Estas son consideradas un factor esencial que ha permitido a las empresas, entre otros logros, llevar a cabo procesos de internacionalización. Esto implica identificar oportunidades de mercado, reducir costos de insumos y mano de obra, disponer de materias primas, medios de trasporte y comunicación, poseer conocimiento de frontera, talentos, flexibilidad de mano de obra, disponibilidad de conocimiento científico, seguridad, respeto a los derechos de propiedad y una economía saludable. De ahí la necesidad de identificar las características más relevantes que deben tener las capacidades tecnológicas.

Aunque múltiples estudios realizados no se han detenido a profundizar en la relación que existe entre las capacidades tecnológicas y la innovación, sí se ha reconocido que estas son un factor de producción esencial y están formadas por un conjunto de conocimientos y habilidades que dan sustento al sistema de producción. Estas identifican y aprovechan las fuentes de aprendizaje y las actividades tecnológicas de las empresas (Westphal, Kritayakirana, Petchsuwan, Sutabutr, \& Ythavong, 1990). De este modo, ellas fomentan el cambio tecnológico, que es una causa fundamental del dinamismo en el mercado y del 
crecimiento económico. Estas habilidades, entre otras cosas, permiten cubrir una serie de requerimientos para iniciar un proceso de mejoras que puede conducir a un sendero de crecimiento y desarrollo sostenido (Lugones, Gutti, \& Le Clech, 2007).

El trabajo de Bell y Pavitt (1993) define las capacidades tecnológicas como los recursos necesarios para generar y gestionar el cambio tecnológico. Para los autores, las capacidades vinculadas con la rutina, como las de producción, por ejemplo, conducen la atención hacia aquellos recursos necesarios para producir eficientemente bienes o servicios innovadores, para generar y gestionar los propios cambios tecnológicos.

La taxonomía de Lall (1992) propone formas de clasificar las capacidades tecnológicas desarrolladas por la empresa con el fin de asimilar, adaptar y mejorar la tecnología obtenida. Establece diferencias, en particular, entre las capacidades de inversión, las de producción y las de soporte. Según este autor, las primeras son las habilidades necesarias para identificar, disponer y conseguir tecnología y personal para el diseño, la construcción y el equipamiento de un nuevo proyecto. Se considera que los costos de capital del proyecto dependen de la escala de producción, la composición del conjunto de bienes producidos, la selección de tecnología y la comprensión que tengan las empresas de las tecnologías implicadas.

Las capacidades de producción, por su parte, según Lall (1992), van desde las habilidades básicas como control de calidad, hasta la operación y el mantenimiento, que son un prerrequisito para otras más avanzadas como la adaptación y la mejora, en donde se empieza a vislumbrar el alcance de resultados innovadores. Innovaciones de mayor alcance, sin embargo, implican un trabajo más profundo en materia de investigación y diseño de nuevos procesos y productos. No se trata solo de desarrollar habilidades que permitan operar y mejorar tecnologías, sino también de efectuar esfuerzos internos para absorber o imitar la tecnología comprada a otras empresas.

Por último, las capacidades de soporte, de acuerdo con el mencionado autor, son las habilidades necesarias para el intercambio de información, tecnología y destrezas entre empresas, proveedores, subcontratistas, consultores, instituciones tecnológicas, universidades, centros de I+D e instituciones gubernamentales. Estas influyen tanto en la eficiencia productiva de las compañías como en la difusión de la tecnología en la industria y pueden desarrollarse en 
distintos niveles. En el nivel primario, la empresa adquiere habilidades básicas, en el nivel medio alcanza habilidades secundarias (es decir, de imitación) y en el nivel más elevado logra las habilidades de innovación más altas.

Las capacidades tecnológicas implican ante todo transmisión de conocimientos entre distintos agentes. Se caracterizan por tener componentes tácitos de conocimiento específico tanto de las personas como de las prácticas internas de las empresas que las generan. Estas se desarrollan a partir de los procedimientos de búsqueda y aprendizaje para mejorar la eficiencia productiva, generar nuevos productos e introducir métodos de organización.

En contraste con los enfoques tradicionales, se señala que el desarrollo tecnológico industrial no debe verse como un proceso que solo se puede promover por medio de la inversión en nuevos equipos y la compra de tecnología importada. Para que la tecnología pueda ser asimilada, bien operada y mejorada, las organizaciones deben realizar inversiones y acciones deliberadas de aprendizaje tecnológico (Bell \& Pavitt, 1995). No basta entonces con comprar máquinas o contratar transferencia de tecnología para reducir la brecha tecnológica internacional. Las em- presas deben investigar sobre nuevas tecnologías, entenderlas y documentarlas para poder asimilarlas, mejorarlas $\mathrm{y}$, eventualmente, crear unas propias.

\subsection{Innovación}

El desarrollo económico de los países desarrollados es distinto al de los emergentes; sin embargo, los dos tipos de naciones comparten algo: ambos se ven obligados a competir en escenarios complejos y sus empresas de manufactura tienen que transformarse para enfrentar las nuevas exigencias que imponen los mercados. Esto demanda que las empresas construyan ventajas estratégicas en innovación de procesos y productos (Bhat, Sushil, \& Jain, 2011; Carayannis \& Campbell, 2012; Teece, 2009). Para ello es necesario que conozcan qué relación existe entre las capacidades tecnológicas y su importancia para competir. Es un hecho, en cualquier caso, que para propiciar la innovación son fundamentales las capacidades tecnológicas. Estas permiten a las empresas adaptarse en distintos escenarios, incluso turbulentos, a fin de lograr una ventaja competitiva sostenible (Zhou \& Wu, 2010).

Múltiples autores definen la innovación desde distintos enfoques. A continuación se mencionan algunos 
de estos conceptos: la innovación puede concebirse como una nueva y original idea que incorpora algún tipo de mejora a otra, a medida que se desarrollan, prueban y aplican aspectos distintos a lo largo del tiempo (Van de Ven, 2001). Mediante esta premisa es clara la relación entre el concepto innovación y el desarrollo de capacidades tecnológicas, desde el diseño hasta la entrega del producto. Zawislakl, Alves y Tello (2012) mencionan que la innovación se entiende como dos procesos de aprendizaje tecnológico de las empresas traducidas en las capacidades de desarrollo de tecnología y operaciones, así como en las rutinas de gestión y transaccionales representadas, justamente, por la capacidad de gestión y transacción.

Du Plessis (2007), por su parte, define la innovación como la creación de nuevos conocimientos (ver Nonaka \& Takeuchi, 1995, 2001) e ideas para facilitar la actividad empresarial, sus resultados, estructuras, procesos, productos y la propia generación de nuevos productos y servicios. Para Dosi (1988) la innovación es resultado de la investigación, el descubrimiento, la experimentación, el desarrollo, la imitación y la adopción de nuevos procesos, nuevos productos y formas de organización de recursos. Así, las empresas hacen un esfuerzo por integrar todas estas estructuras para tener, como ya se indicó, capacidad de respuesta en materia de recursos para la competitividad (Kraatz \& Zajac, 2001).

Tseng (2014) añade que los desafíos de la competencia mundial se reflejan en la fase de la investigación y el desarrollo interno, pero que no siempre pueden ser estos una garantía de éxito para la innovación. Sin embargo, la búsqueda de recursos externos, especialmente de conocimiento tecnológico, se ha convertido en una necesidad para la mayoría de las empresas.

Así, para poder innovar las empresas deben invertir mucho en la construcción de capacidades tecnológicas. Esto significa contar con destrezas y habilidades para utilizar diversos recursos y poder saber cómo hacer las cosas, toda vez que, de acuerdo con Zahra y George (2002), la innovación es primordial para que las empresas puedan adaptarse a los cambios de la economía y el mercado y lograr una ventaja competitiva sostenible.

\subsection{La importancia de la innovación en las empresas}

Parece haber coincidencia entre diversos autores respecto a la importancia de las capacidades tecnológicas para la innovación. Sin embargo, el 
comportamiento de las empresas difiere. Algunos autores, por ejemplo, postulan que las empresas maduras y emergentes exhiben diferentes patrones de crecimiento de capacidades tecnológicas e innovación. Estos tienen efectos en el rendimiento diferencial presente para estos dos tipos de organizaciones ( $\mathrm{Ju}$, Zheng Zhou, Yong Gao \& Lu, 2013).

Los diferentes tipos de empresas de manufactura son la columna vertebral en las economías maduras. Esto, dada la generación de empleos técnicos especializados susceptibles de adaptarse a la cambiante economía global. Las compañías requieren contar con ventajas estratégicas en materia de innovación de procesos y productos. Para esto deben desarrollar capacidades tecnológicas en sus sistemas de producción. Autores como Hadjimanolis (2000) resaltan, en este sentido, la existencia de diferencias entre grandes y pequeñas empresas en relación con las posibles ventajas que ambos tipos de organizaciones demuestran en el desarrollo de innovaciones en su producción. Así, las grandes empresas en economías maduras parecen tener marcadas diferencias por sus capacidades de desarrollar innovaciones en industrias intensivas en inversión comparado con las empresas pequeñas que podrían tener más éxito en industrias en donde la mano de obra calificada representa un factor determinante.

Más aún, las grandes empresas de economías maduras y las pequeñas de economías emergentes muestran también diferencias en la asignación de los recursos necesarios para llevar a cabo actividades de innovación. De ahí que algunos estudios defiendan que las fortalezas en materia de innovación de las pequeñas empresas no se basan tanto en la disponibilidad de recursos, al menos de tipo tangible, sino en ciertas tipologías de procedimiento, más vinculadas con sus propios procesos y productos (Vossen, 1998).

Cabe añadir que dichas empresas actúan como generadoras de innovaciones en sus procesos y productos, ya que esto les proporciona ventajas competitivas. Para desarrollar la innovación, una compañía debe antes que nada buscar, identificar y evaluar conocimientos alternativos de diferentes fuentes $\mathrm{y}$, posteriormente, identificar el conocimiento potencialmente útil. La empresa debe transferir luego ese conocimiento, a partir de las fuentes disponibles y buscarque estepermee el conjunto de la organización (Zhou \& Wu, 2010).

Para Candía, Coliñanco, Caro y Hernández (2014) la importancia de socializar los conocimientos por parte 
de la empresa radica en que la innovación y la gestión de la misma son parte de una estrategia fundamental para el crecimiento de sus negocios. Estos autores añaden que sin innovación estas organizaciones habrían presentado dificultades respecto de su actual posicionamiento en los mercados en donde se desenvuelven.

Por consiguiente, la empresa debeadquirir y transformar el conocimiento en diseños de procesos específicos que posibiliten que un producto sea resultado de la innovación. Las organizaciones requieren producir, innovar, administrar e introducirse provistas de estos elementos en el mercado. Esto se relaciona con cada uno de los tipos de capacidades tecnológicas, tales como la inversión, la producción, la vinculación, la fabricación y la adaptación de maquinaria y equipo (Araiza Garza, Velarde López, \& Chávez Rangel, 2014).

\subsection{Factores de innovación}

Respecto a los factores de innovación, los aportes de diversos autores han tenido como propósito identificar aquellos que influyen en la mejora de innovaciones por parte de las empresas. En contraste con lo mencionado previamente, los argumentos suelen centrarse en grandes empresas de economías maduras. Esto a pesar de que por tradición las principales innovaciones en estas empresas suelen asociarse a las empresas transnacionales, aunque ellas lleguen a estar ubicadas en el contexto de economías emergentes (Vossen, 1998).

Así, a pesar de los esfuerzos que vienen realizando las pequeñas y medianas empresas para atender las sugerencias arriba planteadas, estas deben contemplar nuevas direcciones en materia de investigación, aún a pesar de que se vean limitadas para invertir recursos en proyectos de innovación. Esto dado que necesitan dar cabida a las complejas conexiones entre sus estructuras interna y externa. Ellas, en especial, deben evaluar los procesos de gestión que miden y permiten planear y ejecutar estrategias hacia el futuro (Edwards, Delbridge, \& Munday, 2005).

Diversos estudios consideran que el desarrollo de innovaciones en la industria puede determinar el tipo de procesos de innovación. Esto constituye un cambio para las empresas en I+D (Lichtenthaler, 2008) y refleja una relación positiva entre la innovación de las empresas y los gastos en $\mathrm{I}+\mathrm{D}$ en el sector en el que se opera.

Las empresas invierten en la investigación básica, orientando innovaciones más complejas mediante el uso de alta tecnología. Esto lleva a 
considerar que tienen una avanzada capacidad tecnológica, en otras palabras, una mayor capacidad para la innovación. Merritt (2007), sin embargo, estima que las empresas con mayor actividad interna de $\mathrm{I}+\mathrm{D}$ se pueden beneficiar si establecen una colaboración con las universidades y con los centros de capacitación tecnológica profesional.

De hecho, hay quienes consideran que las empresas deben invertir en estructuras formales que cuenten con personal dedicado a la $\mathrm{I}+\mathrm{D}$ en la exploración de nuevas formas de producción y nuevos productos (Reichert et al., 2011). Existe una serie de factores que inciden en la innovación, entre los que se destacan la I+D en el proceso innovador y el ciclo de vida de la tecnología y del mercado.

En sectores emergentes las empresas tienden a preferir la utilización de fuentes externas de innovación. Estas suelen requerir conocimientos específicos sobre los diferentes sectores, por lo que sus ideas y oportunidades tecnológicas suelen estar relacionadas con el comportamiento del mercado. Ciertamente, los canales de búsqueda pueden proporcionar ideas y recursos que ayudan a las empresas a obtener y explotar oportunidades innovadoras (Laursen \& Salter, 2006).
Las innovaciones también pueden derivar de una red de colaboración entre actores de los sistemas de innovación en cualquier nivel. Pero en sectores de acelerado crecimiento se obstaculiza el aprovechamiento de los recursos internos de las empresas implicadas en la innovación de productos mediante el registro de patentes (Gooroochurn \& Hanley, 2007).

Algunos autores sostienen, por otro lado, que la formación académica de quienes administran una empresa es un factor decisivo para la generación de innovaciones. Estos deben tener una mayor capacidad innovadora, soportada en el funcionamiento de un área especializada en investigación (Levenburg \& Magal, 2006).

Por su parte, Hausman (2005), en un sentido similar, identifica la existencia de una relación positiva entre la innovación de las empresas y el nivel de estudios, en particular, de los responsables financieros. Observa que las personas que tenían una educación más limitada resultaban ser administradores menos innovadores, además explica por qué algunas empresas tienen éxito y otras no, en función de su capital intelectual, el cual fomenta la innovación en procesos y productos.

Carlile (2004) sugiere que las empresas deben utilizar y transformar 
el conocimiento en diseños de bienes y servicios específicos que constituyen, finalmente, el producto innovador. Un factor adicional identificado, que es considerado también como un determinante de la innovación en la empresa es, por último, la pertenencia a redes de cooperación empresarial y entre países (Tseng, 2014). Esto implica que las empresas necesitan contar con las capacidades tecnológicas necesarias para poder llevar a cabo proyectos conjuntos de investigación.

\section{METODOLOGÍA}

En esta investigación se hizo una revisión y un análisis de la literatura especializada considerando los aportes de múltiples autores relevantes (Machi \& McEvoy, 2009). Se contrastaron, además, argumentos existentes sobre empresas en economías maduras con algunos que consideran la realidad de organizaciones en economías emergentes.

\section{RESULTADOS MÁS DESTACADOS}

Aunque en la sección 1 de este documento se han presentado los resultados globales de la revisión de literatura realizada, en este apartado se presentan los resultados tal vez más destacados y se examinan de manera sintética los aportes de los autores probablemente más relevantes que han dedicado sus esfuerzos a estudiar las capacidades tecnológicas en relación con la innovación.

Araiza Garza et al. (2014) señalan que las empresas que cooperan entre sí logran desarrollar niveles más altos de capacidades que las que no lo hacen, por lo tanto, la cooperación entre empresas puede ser utilizada como una estrategia que permite desarrollar las capacidades tecnológicas, haciendo a las compañías cada vez más competitivas. Al igual que otros conceptos, las capacidades tecnológicas han tenido una serie de acepciones diferentes a lo largo del tiempo y se han visto influidas por la coyuntura económica del momento y las circunstancias por las que atraviesa cada país. Sin embargo, también ha incidido el contexto en la forma como se desarrollan y organizan dichas capacidades. Las diferencias en los enfoques han enriquecido el análisis al respecto y permiten hoy contrastar los argumentos a lo largo del tiempo.

Los aportes de algunos autores, como Reichert et al. (2011), reflejan la importancia de la innovación y de las capacidades tecnológicas para el desarrollo económico, ya sea en la generación de nuevos procesos, nuevos productos o nuevas oportunidades de mercado. En la literatura 
especializada se han relacionado las coincidencias de clasificar el nivel tecnológico en producción y procesos de las empresas según el sector al que pertenecen. En esta categorización la manufactura usual industrial depende regularmente de sus proveedores en relación con el cambio tecnológico. Según los autores analizados, industrias de este tipo más a menudo hacen mejoras y los cambios en los métodos de producción y diseño de productos, en lugar de transformaciones radicales, ya que la transferencia tecnológica sucede fácilmente porque surge de los proveedores del equipo (Bell \& Pavitt, 1995).

Para estudios de contraste se considera que la innovación está relacionada con el proceso de reunir conocimientos y recursos suficientes para alcanzar un cambio importante en las empresas. Los argumentos giran en torno a proteger y adaptar tecnologías a las circunstancias competitivas. Las compañías ganan y generan conocimiento haciendo pequeñas innovaciones precisas para apoyar su funcionamiento y desarrollo. Al respecto, Freeman y Soete (2007) reconocen que la competencia se basa cada vez más en la innovación. Argumentan que este es un reto importante para la capacidad tecnológica en países en economías maduras.
Simultáneamente, la tecnología y la innovación pueden tener una significativa relación con el estudio de la capacidad tecnológica de una empresa. Por lo anterior, hay una gran diferencia en los estudios sobre el tema, pero se aprecian, en particular, diferencias cuando se habla de empresas en economías maduras respecto a los estudios relativos a compañías ubicadas en economías emergentes (Reichert et al., 2011).

\section{DISCUSIÓN Y CONCLUSIONES}

Las capacidades tecnológicas son un proceso evolutivo, fuente de inspiración de las estrategias de las grandes empresas maduras en cuanto a su flexibilidad en sus sistemas de producción. Augier y Teece (2006) consideran que el desempeño de una empresa se ve afectado por su capacidad para construir continuamente, combinar, integrar y reordenar los recursos y competencias.

Ellonen, Wikström y Jantunen (2009) mencionan que el éxito en la innovación está influido por la relación entre la experiencia en el mercado y la experiencia tecnológica acumulada por una empresa. De manera que la tecnología y la innovación pueden ser vistas como teniendo una significativa relación con el estudio de la capacidad tecnológica de una organización. 
Las capacidades tecnológicas son un conjunto de habilidades dinámicas y cambiantes. Estas son esenciales para el uso efectivo del conocimiento científico y tecnológico disponible y, por ende, se han convertido en un recurso vital para generar innovaciones en las empresas.

Las empresas pueden ser cada vez más competitivas en la medida en que mejoren sus actividades internas y aprovechen sus capacidades tecnológicas, en especial aquellas que permiten solucionar problemas asociados al mercado y la comercialización, esto es, tener una capacidad de respuesta a los cambios del entorno competitivo.

Se considera que las actividades de soporte en los países emergentes requieren una plataforma que incluya, entre otros aspectos, información técnica, económica y estratégica que dé cuenta de la actualidad más relevante para la compañía. Dichas actividades facilitan asimismo los diseños de nuevos tipos de procesos y productos, a partir de la trasferencia de tecnología en los diferentes sectores de la industria.

Se infiere que la colaboración en proyectos tecnológicos con las universidades y con otras empresas, tomando como base la experiencia de los países con economías maduras, se traduce en resultados positivos para las organizaciones. La disposición de 1) participar con los centros de I+D, 2) realizar proyectos tecnológicos con las instituciones gubernamentales, 3) hacer parte de proyectos de colaboración interempresariales o con centros de capacitación tecnológica profesional y 4) realizar otras acciones similares permite apalancar los procesos destinados a generar innovaciones.

La conjunción de los tres conceptos estudiados: inversión, producción y soporte, es determinante. El éxito de identificar factores determinantes de innovación varía en función de múltiples factores y circunstancias, como el nivel de desarrollo económico de los países, el tipo de empresas, el tamaño de las mismas, el sector al que pertenecen, sus exigencias tecnológicas y el tipo de procesos de manufactura implementado en la integración conjunta de todas sus actividades.

Finalmente, es necesario percatarse de que las capacidades tecnológicas juegan un papel esencial para el desarrollo económico, científico y tecnológico de los países, en particular, de los emergentes. De ahí la importancia de identificar las actividades de las empresas de un país. Esto puede contribuir a diseñar estrategias específicas de respaldo que 
faciliten a las compañías la tarea de identificar nuevas oportunidades de innovación, lo que les puede permitir desarrollar las capacidades tecnológicas que necesitan para ser más competitivas en los mercados, generar cada vez más innovaciones $\mathrm{y}$ tener una mejor capacidad de respuesta. La capacidad de respuesta de una organización manufactura, en particular, es un tema que debe ser tomado en cuenta en futuras investigaciones. Esto para poder establecer si se trata de un concepto diferente al de capacidades tecnológicas para la innovación o es, más bien, probablemente, un producto de estas.

\section{REFERENCIAS}

Acosta-Prado, J. C., Bueno Campos, E., \& Longo Somoza, M. (2014). Technological capability and development of intellectual capital on the new technologybased firms. Cuadernos de $\mathrm{Ad}$ ministración, 27(48), 11-39.

Araiza Garza, Z., Velarde López, E., \& Chávez Rangel, M. (2014). La cooperación interempresarial y su relación con el desarrollo de las capacidades tecnológicas en las pymes de la industria metalmecánica de la región centro de Coahuila, en México. Revista Internacional Administración \& Finanzas, 7(2), 13-29.
Augier, M., \& Teece, D. J. (2006). Understanding complex organization: The role of know-how, internal structure, and human behavior in the evolution of capabilities. Industrial and Corporate Change, 15(2), 395-416. Augier, M., \& Teece, D. J. (2008). Strategy as evolution with design: The foundations of dynamic capabilities and the role of managers in the economic system. Organization Studies, 29(8/9), 1187-1208.

Barney, J. (1991). Firms resources and sustain competitive advantage. Journal of Management, 17(1), 99-120.

Barney, J. B., \& Clark, D. N. (2007). Resource-based theory: Creating and sustaining competitive advantage. Oxford - Nueva York: Oxford University Press.

Bell, M., \& Pavitt, K. (1993). Technological accumulation and industrial growth: Contrasts between developed and developing countries. Industrial and Corporate Change, 2(2), 157-211.

Bell, M., \& Pavitt, K. (1995). The development of technological capabilities. En R. Irfan-ul-Haque \& M. N. Bell (Eds.) Trade, technology, and international competitiveness (pp. 69-101). Washington: The World Bank.

Benner, M., \& Tushman, M. (2003). Exploitation, exploration and pro- 
cess management: The productivity dilemma revisited, Academy of Management Review, 28(2), pp. 238-256.

Bhat, J. S. A., Sushil, \& Jain, P. K. (2011). Innovation by harmonizing continuity and change. The Journal of Business Strategy, 32(2), 38-49.

Candía, J. G., Coliñanco, L. G., Caro, C. L., \& Hernández, N. R. (2014). Estrategia y cultura de innovación, gestión de los recursos y generación de ideas: prácticas para gestionar la innovación en empresas. Pensamiento \& Gestión, 36, 107-133.

Carayannis, E. G., \& Campbell, D. F. J. (2012). Mode 3 knowledge production in quadruple helix innovation systems: $21^{\text {st }}$-century democracy, innovation, and entrepreneurship for development. Nueva York - Londres: Springer.

Carlile, P. R. (2004). Transferring, translating, and transforming: An integrative framework for managing knowledge across boundaries. Organization Science, 15(5), 555-568.

Chung, W., \& Swink, M. (2009). Patterns of advanced manufacturing technology utilization and manufacturing capabilities. Production and Operations Management, 18(5), 533-545.

Combs, J. G., Ketchen Jr, D. J., Ireland, R. D., \& Webb, J. W.
(2011). The role of resource flexibility in leveraging strategic resources. Journal of Management Studies, 48(5), 1098-1125.

Dosi, G. (1988). Technical change and economic theory. Nueva York: Pinter Publishers.

Du Plessis, M. (2007). The role of knowledge management. Journal of Knowledge, 11(4), 20-29.

Dutrenit, G., \& Capdevielle, M. (1993). El perfil tecnológico de la industria mexicana y su dinámica innovadora en la década de los ochenta. El Trimestre Económico, 60(239). 643-674.

Dutrenit, V. C., \& Arias. (2002). Diferencias en el perfil de acumulación de capacidades tecnológicas en tres empresas mexicanas. El Trimestre Económico, 70(277), 109-165.

Edwards, T., Delbridge, R., \& Munday, M. (2005). Understanding innovation in small and mediumsized enterprises: A process manifest. Technovation, 25, 11191127.

Ellonen, H. K., \& Jantunen, A. (2011). The role of dynamic capabilities in Developing innovation-related capabilities. International Journal of Innovation Management, 15(3), 459-478.

Ellonen, H., Wikström, P., \& Jantunen, A. (2009). Linking dynamiccapability portfolios and innovation. Technovation, 29(11), 753762. 
Etzkowitz, H. (2008). The triple helix: University-industry-government innovation in action. Nueva York: Routledge.

Figueiredo, P. N. (2004). Aprendizagem tecnológica e inovação industrial em economias emergentes: uma breve contribuição para o desenho e implementação de estudos empíricos e estratégias no Brasil. Revista Brasileira de Inovação, 2(3), 323-361.

Freeman, C., \& Soete, L. (2007). Developing science, technology and innovation indicators: What we can learn from the past. Research Policy, 6(1), 583-589.

Gibbs, R., \& Humphries, A. (2009). Strategic alliances \& marketing partnerships: Gaining competitive advantage through collaboration and partnering. Londres - Philadelphia: Kogan Page.

González, R. K., \& Cunha, S. K. (2012). Internationalization process and technological capability trajectory of Iguaçu. Journal of Technology Management \& Innovation, 7(2), 117-129.

Gooroochurn, N., \& Hanley, A. (2007). A tale of two literatures: Transaction costs and property. Research Policy, 36(10), 14831495.

Hadjimanolis, A. (2000). An investigation of innovation antecedents in small firms in the R\&D. Management, 30(3), 235-245.
Hausman, A. (2005). Innovativeness among small businesses: Theory and propositions for future research. Industrial Marketing Management, 34(8), 773-782.

Ju, M., Zheng Zhou, K., Yong Gao, G., \& Lu, J. (2013). Technological capability growth and performance outcome: Foreign versus local firms in China. Journal of International Marketing, 21(2), 1-16.

Kraatz, M. S., \& Zajac, E. J. (2001). How organizational resources affect strategic change and performance in turbulent environments: Theory and evidence. Organization Science, 12(5), 632657.

Lall, S. (1992). Technological capabilities and industrialization. World Development, 20(2), 165186.

Laursen, K., \& Salter, A. (2006). Open for innovation: The role of openness in explaining innovation performance among U.K. manufacturing firms. Strategic ManagementJournal,27(2), 131150.

Levenburg, N., \& Magal, S. (2006). An exploratory investigation of organizational factors and e-business motivations among SMFOEs in the US. Electronic Markets, 16(1), 70-84.

Lichtenthaler, U. (2008). Open innovation in practice: An analysis 
of strategic approaches to technology transactions. IEEE Transactions on Engineering Management, 64(4), 149-160.

Lugones, G. E., Gutti, P., \& Le Clech, N. (2007). Indicadores de capacidades tecnológicas en América Latina. México: CEPAL, Unidad de Comercio Internacional e Industria.

Machi, L. A., \& McEvoy, B. T. (2009). The literature review: Six steps to success. Thousand Oaks - Londres: Corwin.

Madhok, A., Li, S., \& Priem, R. L. (2010). The resource-based view revisited: Comparative firm advantage, willingness-based isolating mechanisms and competitive heterogeneity. European Management Review, 7(2), 91-100.

Merritt, H. (2007). La vinculación industria-centros tecnológicos de investigación y desarrollo: el caso de los centros CONACYT. Análisis Económico, 12(49), 149-168.

Nelson, R., \& Winter, S. (1982). An evolutionary theory of economic change. Cambridge:HarvardUniversity Press.

Nonaka, I., \& Takeuchi, H. (1995). The knowledge-creating company: How Japanese companies create the dynamics of innovation. Nueva York: Oxford University Press.

Nonaka, I., \& Takeuchi, H. (2001). Facilitar la creación de cono- cimiento. México: Oxford University Press.

Organización para la Cooperación y el Desarrollo Económico (OECD) (2005). Directorate for science, technology and industry. París: Organization for Economic CoOperation and Development.

Pavitt, K. (1984). Sectoral patterns of technical change: Towards a taxonomy and a theory. Research Policy, 13(6), 343-373.

Reichert, F. M., Beltrame, R. S., Corso, K. B., Trevisan, M., \& Zawislak, P. A. (2011). Technological capability's predictor variables. Journal of Technology, 6(1), 14-25.

Schilirò, D. (2012). Knowledge-based economies and the institutional environment. Theoretical \& Practical Research in Economic Fields, 3(1), 42-50.

Teece, D. J. (2008). Explicating dynamic capabilities: The nature and microfoundations of (long run) enterprise performance. En M. Augier \& D. J. Teece (Eds.). Fundamentals of business strategy (Vol. 5, pp. 263-301). Los Ángeles - Londres: SAGE.

Teece, D. J.(2009).Dynamic capabilities and strategic management: Organizing for innovation and growth. Oxford - Nueva York: Oxford University Press.

Teece, D. J., Pisano, G., \& Shuen, A. (1997). Dynamic capabilities 
andstrategicmanagement.Strategic Management Journal, 18(7), 509-533.

Tseng, C. Y. (2014). Technological innovation capability, knowledge sourcing and collaborative innovation in gulf cooperation council countries. Innovation, 16(2), 212-223.

Van de Ven,A.P.(2001)El viaje de la innovación: el desarrollo de una cultura organizacional para innovar. México: Oxford University Press.

Vossen, R. (1998). Relative strengths and weaknesses of small firms in innovation. International Small Business Journal, 16(3), 88-94.

Westphal, L. E., Kritayakirana, K., Petchsuwan, K., Sutabutr, H., \& Ythavong, Y. (1990) The development of technological capability in manufacturing: macroscopic approach to policy research. En R. Evenson \& G. Ranis (Eds.). Science and technology: Lessons for development policy (pp. 81134), Londres: Intermediate Technology Publications.

Wilden, R., Gudergan, S., \& Lings, I. (2011). Understanding the market in turbulent times: The impact of opportunity identification and reconfiguring on capabilities
(Ponencia presentada a la AMA Summer Educators' Conference, San Francisco, Estados Unidos). Winter, S. (2008). Understanding dynamic capabilities. En M. Augier \& D. J. Teece (Eds.). Fundamentals of business strategy (Vol. 5, pp. 217-222). Los Ángeles - Londres: SAGE.

Yokakul, N., \& Zawdie, G. (2010). Innovation network and technological capability development in the Thai SME sector: The case of the Thai dessert industry". International Journal of Technology Management \& Sustainable Development, 9(1), 19-36.

Zahra, S. A., \& George, G. (2002). Absorptive capacity: A review, reconceptualization, and extension. The Academy of Management Review, 27(2), 185-203.

Zawislak, P. A., Alves, A. C., \& Tello-Gamarra, J. (2012). Innovation Capability: From technology development to transaction capability. Journal of Technology Management \& Innovation, 7(2), 14-26.

Zhou, K. Z., \& Wu, F. (2010). Technological capability, strategic flexibility, and product innovation. Strategic Management Journal, 31(5), 547-561. 